\section{NP25 Talking to Parents of Middle School Children About How to Support Healthful Food Choices at Home and at School}

Tisa Hill, MPH, tfh3@cornell.edu, Cornell University, Division of Nutritional Sciences, 343 Martha Van Rensselaer Hall, Ithaca, NY 14853; T. F. Hill, MPH; A. Gaines, PhD; E. Green, RD; L. N. Thomas, PhD; D. R. Just, PhD; B. Wansink, PhD; J. S. Dollahite, PhD

Objective: To develop effective nutrition messages with complementary strategies for parents of middle school children that will positively impact children's food choices.

Description: Researchers conducted 7 focus groups with 46 mothers of middle school students in urban and rural settings. Utilizing card sorts, participants categorized nutrition behaviors and associated parenting skills by level of perceived importance. Ease or difficulty of putting behaviors into practice was assessed using open-ended questions. Results informed development of messages and strategies addressing parenting skills and core nutrition practices (increasing fruit, vegetable, and low-fat dairy intake, and decreasing sweetened beverage intake). Researchers then conducted semi-structured interviews, during which 24 parents ranked messages by preference and selected most relevant strategies to affect children's behaviors.

Evaluation: Focus group transcripts were open-coded to identify nutrition-related behaviors of concern and important parenting strategies. Message ranking and strategy selection were examined for frequency, while interview transcripts were reviewed to explain choices and edit content using participant suggestions.

Conclusions and Implications: Parents preferred simple, direct information that reinforced parenting roles. Highest ranked messages promoted fruit and vegetable variety, supported role modeling, and promoted water as a preferred beverage. Parents expressed interest in strategies for engaging children in food selection and preparation and strategies for healthful snacks and meals. These results provide valuable insight from parents of middle school students - an underrepresented group of adolescents in transition - and will inform future messaging to parents.

Funding: USDA Grant \#2012-68001-19604

\section{NP26 Use of Engaging Online Videos in Conjunction With New Feeding Content to Enhance a Current EFNEP Program}

Sheryl Hughes, PhD, shughes@bcm.edu, Baylor College of Medicine, 1100 Bates, Houston, TX 77030; T. Power, PhD, Washington State University; S. Baker, EdD, Colorado State University; K. Barale, MS, Washington State University; J. Lanigan, PhD; L. Parker, PhD

Objective: Researchers have posited that inclusion of the family may be necessary in childhood obesity prevention efforts. Parenting practices that thwart children's ability to pay attention to internal cues of hunger and fullness may contribute to childhood obesity. The overall purpose of this study is to add parenting content to the widely used Eating Smart * Being Active Expanded Food and Nutrition Education Program. This will be accomplished by modifying previously developed videos for use in the parenting curriculum which will provide parents with appropriate tools to use in feeding their children.

Description: Participants will be mothers who are participating in the Eating Smart Being Active EFNEP nutrition program in the states of Colorado and Washington. Parenting curriculum will be delivered in addition to the nutrition program in one of two conditions - online versus in-person.

Evaluation: Currently, the parent curriculum is being developed along with the website and activities for use in the online condition and activities for the in-person condition. Modifications to the existing video recordings have been made and additional footage produced. Next steps will include piloting the program. Modifications will be made based on the pilot prior to commencing the randomized controlled trial. Evaluations to the program will take place at pre-, post-, at 6 and 12 months.

Conclusions: Childhood obesity prevention efforts have rarely been successful. Incorporating parents in prevention efforts may be a missing link in combatting childhood obesity.

Funding: USDA Grant \#2015-68001-23311

\section{NP27 Children Eating Well (CHEW) Smartphone Application for WIC-Participating Families With Preschool Children}

Pamela Hull,PhD, pam.hull@vanderbilt.edu, Vanderbilt University School of Medicine, 2525 West End Avenue, Suite 800, Nashville, TN 37203; J. Emerson, PhD,

Tennessee State University; M. Quirk, PhD; D. Schmidt, PhD, Vanderbilt University School of Engineering; $S$. Mulvaney, $P h D$, Vanderbilt University School of Nursing; B. Beech, DrPH, University of Mississippi Medical Center; B. A. Husaini, PhD, Tennessee State University

Objective: The objective was to develop and evaluate a smartphone-based nutrition education intervention that is culturally appropriate for African American and Hispanic WIC participants with preschool children.

Description: Based on findings from the CHEW Nutrition Survey and input from the CHEW Community Advisory Board and WIC program, the team developed a smartphone application with two components: a WIC shopping tool to assist mothers with maximizing WIC vouchers, and nutrition education focused on healthy snacks and beverages targeted for ages 2-4. The goals of the smartphone intervention (English and Spanish) were to make the WIC shopping experience easier, maximize WIC voucher use, and improve parent snack feeding practices. The dietary targets for children were increased intake 


\section{NP27 (continued)}

of fruits/vegetables, water, and low-fat milk, and decreased intake of sugar-sweetened beverages.

Evaluation: The primary analysis examined feasibility and user engagement based on usage, usability, and perceived benefits. Secondary analysis examined preliminary effects of the smartphone app prototype on behavioral outcomes using a two-group randomized controlled design with 3 -month follow up ( $\mathrm{N}=160$ mothers). The application successfully demonstrated feasibility and user acceptance, with specific areas for improvement identified for future versions. Preliminary results suggested improvements in variety of fruits/vegetables in the home and some parent snack feeding practices.

Conclusions and Implications: Since the application was developed using an open source framework, future versions can be adapted and disseminated to WIC programs across the United States.

Funding: USDA Grant \#2011-68001-30113

\section{NP28 Latino Fathers Promoting Healthy Youth Behaviors}

Ghaffar Hurtado,PhD, hurt0033@umn.edu, University of Minnesota, 1420 Eckles Avenue, St. Paul, MN 55108;

K. Shirer, PhD; M. Reicks, PhD; M. Allen, MD

Objective: This integrated Extension and Research project aims to prevent obesity among Latino youth (10-14 years) by engaging fathers and youth in a culturallyand linguistically-appropriate prevention program. Participation is expected to increase father involvement in positive parenting practices to address youth energy balance-related behaviors (EBRBs) (i.e. sugar sweetened beverage, fruit and vegetable, and breakfast consumption, physical activity and sedentary behavior).

Description: An existing parenting curriculum shown to be effective for Latino fathers to prevent youth substance abuse will be adapted using community-based participatory research principles. The 8-week program will address paternal parenting practices related to the food and physical activity environment in the home (role modeling, availability, expectations, communication). In year 1 (beginning March 1, 2016), initial focus group interviews and parent advisory board meetings will be conducted to inform curriculum adaptation, followed by pilot testing. The program will be implemented and evaluated in years 2-4, with final evaluation results analyzed and disseminated in year 5 .

Evaluation: A randomized controlled study with a delayed-treatment condition for the control group will be conducted with randomization at the father-youth pair/ dyad within community agencies $(n=40$ dyads in each of 6 sites). Baseline, immediate post-intervention, and 3 month post-intervention data will be collected to assess changes in paternal parenting practices, youth EBRBs and weight status.

Conclusions and Implications: The project addresses obesity prevention and healthy behaviors for a growing immigrant population in the United States, targeting fathers of early adolescents, to address youth EBRBs.

Funding: USDA Grant \#2015-07962

\section{NP29 Generating Rural Options for Weight Healthy Kids and Communities - Outcomes and Impacts}

Deborah John,PhD, deborah.john@oregonstate.edu, College of Public Health and Human Sciences, Oregon State University, 106 Ballard Hall, Corvallis, OR 97331; K. Gunter, PhD, PD/PI; P. Hystad, PhD; G. Langellotto, PhD; M. Manore, $P h D$

Objective: A stakeholder-informed contextual model for rural obesity prevention emerged from communityengaged participatory research and resident-informed resource maps and readiness conversations collected using our HEAL MAPPS TM Toolkit. Rural communities $(n=3)$ and elementary schools $(n=3)$ were engaged in developing and implementing data-driven environmental (PSE) strategies to change the obesogenic context (GROW); comparison communities $(n=3)$ and schools $(n=3)$ received educational programming during the 3-year intervention study implemented in 3 geographically diverse Oregon counties.

Description: Mixed-method triangulation design was applied longitudinally at multiple levels to assess child-level and family-level intervention effects, and evaluate PSE changes in the school and community context. Environmental changes and intervention exposures in all participating communities were examined in relation to improved weight-healthy behaviors and obesity prevalence (BMI) among elementary school students.

Evaluation: Obesity and physical activity (PA) were inversely associated among elementary students. Food insecure families were more likely to use school meal programs and less likely to provide out-of-school PA opportunities. Improved school and community capacity, resources, and readiness to address obesogenic factors emerged in all communities but more so in GROW intervention sites. Preliminary analyses of primary outcome data - students' height-weight collected biannually over 3 years - indicate that in the GROW sites children's weight health is improving. Increased population exposure options to multiple and various community and school PSE strategies that support children's healthy eating and physical activity behavioral patterns and population weight-health improvements were positively related.

Conclusions and Implications: Rural context is inadequately resourced to easily support weight-healthy patterns for under-resourced families. Improving the encountered obesogenic context at multiple ecological levels is required to support children's weight-healthy patterns over time and address rural obesity disparities.

Funding: USDA Grant \#2011-68001-30020 